\title{
Wiring Edge-Disjoint Layouts ${ }^{\star}$
}

\author{
Ruth Kuchem ${ }^{1}$ and Dorothea Wagner ${ }^{2}$ \\ 1 RWTH Aachen \\ 2 Fakultät für Mathematik und Informatik, Universität Konstanz, \\ 78434 Konstanz, Germany
}

\begin{abstract}
We consider the wiring or layer assignment problem for edgedisjoint layouts. The wiring problem is well understood for the case that the underlying layout graph is a square grid (see [8]). In this paper, we introduce a more general approach to this problem. For an edgedisjoint layout in the plane resp. in an arbitrary planar layout graph, we give equivalent conditions for the $k$-layer wirability. Based on these conditions, we obtain linear-time algorithms to wire every layout in a trihexagonal grid, respectively every layout in a tri-square-hexagonal grid using at most five layers.
\end{abstract}

\section{Introduction}

The wiring problem consists in converting a two-dimensional edge-disjoint layout into a three-dimensional vertex-disjoint layout. Wiring edge-disjoint layouts is a fundamental and classical problem in VLSI-design. Typically, the general layout problem in VLSI-design consists of two phases, the placement and the routing. Often, the routing phase is again divided into two steps. First, a twodimensional layout is constructed satisfying certain conditions induced by the underlying layout model. This layout describes the course of the wires connecting the corresponding terminals. In the second step, the wiring step or layer assignment step, the edges of the wires are assigned to different layers to avoid physical contacts between different wires. The layout can be viewed as a projection in the plane of this final three-dimensional wiring. In connection with graph drawing, the wiring problem is of interest as well. There, an edge-disjoint embedding of a graph is given. The problem consists in a visualization of the graph by a three-dimensional vertex-disjoint embedding whose projection in the plane is again the edge-disjoint embedding of the graph.

Consider an edge-disjoint layout in the plane resp. in a planar graph. Such a layout may be an edge-disjoint realization of nets or an edge-disjoint embedding of a graph. The construction of edge-disjoint layouts is a fundamental problem. For an overview we refer to $[3,9,11]$. Then the wiring problem can be described as follows. There is a number of graphs isomorphic to the layout, called layers. Each path, called wire, of the layout is realized by a sequence of subpaths in different

* The second author acknowledges the $D F G$ for supporting this research under grant Wa $654 / 10-2$. 
layers such that two different wires are vertex-disjoint. A vertical connection between layers, a via, is used at each layer change. The main optimization goal is to minimize the number of layers.

Several results have been obtained for the wiring problem for edge-disjoint layouts in regular grids, especially square grids $[1,2,4,5,6,10,12,15,14,16]$. Most of these results are based on the combinatorial framework introduced in [8] which applies to edge-disjoint layouts in square grids. Moreover, only layouts where terminals are placed on the boundary of the layout graph are considered. A technique using two-colorable maps is developed, and necessary and sufficient conditions for the wirability in a fixed number of layers are given. For two layers these conditions are easy to test. On the other hand, it is $\mathcal{N} \mathcal{P}$-complete to decide if a layout is three-layer wirable [7]. Every layout is wirable in four layers, and such a wiring can be constructed in time linear in the size of the layout [1], [14]. In [14], the concept of two-colorable maps is applied to octo-square grids, but no guarantee for the number of layers required for the wiring is given. If the layout graph is a tri-hexagonal grid, every edge-disjoint layout is wirable in five layers [13].

Nothing is known so far about the wiring problem for layouts in the plane resp. in general planar layout graphs, with arbitrary terminal positions. In this paper, we develop a general approach to this problem. It leads to necessary and sufficient conditions for $k$-layer wirability of edge-disjoint layouts where at most two different wires meet in a vertex. These conditions generalize the framework given in [8]. Again, two-layer wirability is easy to test. And of course, deciding three-layer wirability is $\mathcal{N} \mathcal{P}$-complete as well. For layouts in special planar layout graphs guarantees are given for the number of layers required for the wiring. We prove that every layout in a tri-hexagonal grid is wirable in at most five layers. Moreover, every layout in a tri-square-hexagonal grid is wirable in at most five layers as well. In both cases, such a wiring can be constructed in time linear in the size of the layout. Observe that our approach for layouts in tri-hexagonal grids is different from the approach given in [13].

The wiring theory presented here is restricted to layouts where at most four wire edges are incident to the same vertex. But it is extendable to the case that more than four wire edges belonging to at most two different wires are incident to the same vertex. The case that more than two different wires meet at a vertex seems to be much more involved.

The paper is organized as follows. In Section 2, we introduce the necessary definitions and notations. The general approach to wiring edge-disjoint layouts is developed in Section 3. Finally, in Section 4 we present linear-time algorithms for wiring layouts in tri-hexagonal and tri-square-hexagonal grids using at most five layers.

\section{Preliminaries}

We consider an edge-disjoint layout in the plane or in a planar layout graph. Such a layout in the plane consists of vertices, called terminals, in the plane and 
pairwise edge-disjoint Steiner trees connecting specified sets of terminals, called wires. A special case of an edge-disjoint layout is an edge-disjoint embedding of a graph, i. e., a mapping of the vertices of that graph into the plane and a realization of its edges by pairwise edge-disjoint paths. Similarly, for an edge-disjoint layout in a planar graph consider an undirected graph $G$, the layout graph, with a fixed embedding in the plane. Then the layout consists of terminals placed on vertices of $G$ and pairwise edge-disjoint Steiner trees connecting specified sets of terminals, respectively pairwise edge-disjoint paths realizing edges of the embedded graph. A layout in the plane, respectively the vertices and edges of the layout graph $G$ occupied by a layout, induce a planar embedded graph. In the following, we identify a layout $L$ with this induced graph.

A conducting layer, or simply layer is a graph isomorphic to the layout $L$. Conducting layers $L_{0}, \ldots, L_{k-1}$ are assumed to be stacked on top of each other, with $L_{0}$ on the bottom and $L_{k-1}$ on the top. A contact between two layers, called a via, can be placed only at a vertex of a layer.

A correct layer assignment or wiring $W(L)$ of a given layout $L$ is a mapping of each edge of $L$ to a layer such that:

1. No two different wires share a vertex on the same layer.

2. If adjacent edges of a wire are assigned to different layers, a via is established between these layers at their common vertex.

3. If a via connects $L_{h}$ and $L_{j}(h<j)$, then layers $L_{i}, h<i<j$, are not used at that vertex by any other wire.

Note that a correct wiring can be interpreted as a three-dimensional configuration of vertex-disjoint wires. In the following, we restrict to layouts where at most two different Steiner trees meet at the same vertex. The case that more than two different wires meet at a vertex seems to be much more involved. Because of lack of space, the wiring theory presented here is formulated only for layouts where at most four wire edges are incident to the same vertex. But it is extendable to the case that more than four wire edges belonging to at most two different wires are incident to the same vertex. To determine a correct wiring of a layout $L$, only those vertices where two different wires meet are of relevance. In the following we call these vertices non-trivial vertices of the layout. In Figure 1, all possibilities of non-trivial vertices where at most four wire edges meet are shown.

Definition 1. The subgraph of $L$ induced by all non-trivial vertices and all edges incident to at least one non-trivial vertex of $L$ is called the core of $L$, denoted $\operatorname{core}(L)$.

The following lemma shows that we can restrict to the core of a layout $L$. In $[8]$, it is proved for layouts in grids, where different wires may cross or both bend at the same vertex, but do not meet at terminals. But the proof of the lemma applies to the more general layouts we consider here as well.

Lemma 2. [8] A layout $L$ is wirable in $k$ layers if and only if each connected component of core $(L)$ is. 


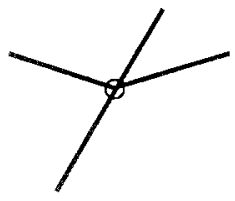

a)

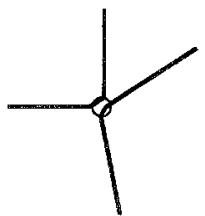

b)

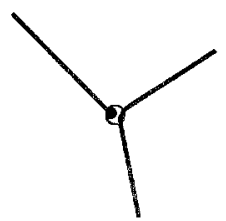

c)

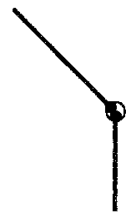

d)

Fig. 1. The different possibilities of non-trivial vertices. Each of the two wires may end at a terminal in that vertex. Possibility c) occurs when one wire ends at a terminal and d) occurs when both wires end at a terminal in that vertex.

Proof. In order to prove the non-trivial part of the lemma, consider a $k$-layer wiring $W$ of the core core $(L)$. All edges of $L$-core $(L)$ are assigned to an arbitrary layer, say $L_{0}$. If necessary, a via is established on a trivial vertex connecting the wires incident to that vertex.

\section{A characterization of $k$-layer wirability}

In this section, we develop a complete characterization of $k$-layer wirability of planar layouts. First, we observe that we can restrict to the 2-edge-connected components of a layout core.

Lemma 3. A layout $L$ is wirable in $k$ layers if and only if each 2-edge-connected component of core $(L)$ is wirable in $k$ layers.

Proof. In order to prove the non-trivial part of the lemma, consider a decomposition of core $(L)$ into its 2-edge-connected components. These 2-edge-connected components can be ordered topologically. Let $W$ be a $k$-layer wiring of the 2edge-connected components, and $P$ be a wire going through different components. Then there is an edge $\{u, v\}$ on $P$ such that $u$ and $v$ belong to different components, say $C(u)$ and $C(v)$. Assume w.l.o.g. that $C(u)$ is before $C(v)$ in the topological ordering.

Now, a correct $k$-layer wiring $W^{*}$ of the subgraph of core $(L)$ induced by $C(u),\{u, v\}$ and $C(v)$ is constructed as follows. In case the wirings of $C(u)$ and $C(v)$ are compatible, i. e., $P$ is wired both times above (resp. below) the wire it meets in $u$ and in $v$, the wiring of $C(v)$ and $C(u)$ remains fixed, $\{u, v\}$ is wired in the same layer as $P$ in $u$, and an appropriate via is placed on $v$. Otherwise, only the wiring of $C(u)$ remains fixed and the wiring of $C(v)$ is flipped. That is, if an edge of $C(v)$ belongs to layer $L_{i}, 0 \leq i \leq k-1$ in $W$, then this edge is assigned to layer $L_{k-i}$ in $W^{*}$. Then again, $\{u, v\}$ is wired in the same layer as $P$ in $u$, and an appropriate via is placed on $v$. Finally, a sequence of flippings according to an arbitrary linear extension of the topological ordering of the 2-edge-connected components of core $(L)$ induces a correct layer assignment of $L$ in $k$ layers. 
In the following, we assume that core $(L)$ is 2-edge-connected. The dual graph of a planar graph with respect to a fixed combinatorial embedding is defined as follows. For each face of the graph there is a dual vertex, and there is an edge connecting two dual vertices if and only if their faces are incident with a common primal edge. The subgraph of the dual graph of $L$ corresponding to core $(L)$ is denoted by core $(L)^{d}$. That is, core $(L)^{d}$ is the graph induced by all edges dual to edges of core $(L)$. Since core $(L)$ is 2-edge-connected, core $(L)^{d}$ contains no loops. The edges of core $(L)^{d}$ that are dual to edges of core $(L)$ incident to a trivial vertex are called the boundary edges of core $(L)^{d}$, or just the boundary of core $(L)^{d}$. Vertices of core $(L)^{d}$ incident to boundary edges are called boundary vertices, all other vertices of core $(L)^{d}$ are called inner vertices. See Figure 3. Obviously, a vertex is an inner vertex if and only if it is dual to a face of core $(L)$ that contains only non-trivial vertices.
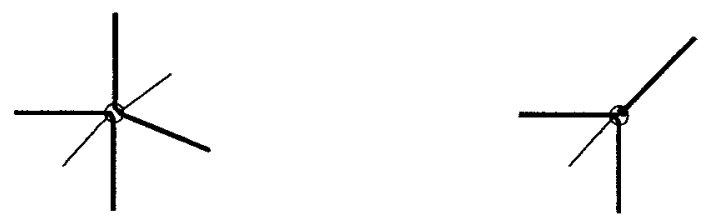

Fig. 2. Diagonals (thin) corresponding to a pair of neighbored edges of core $(L)$ that belong to the same wire.

We first give a characterization of two-layer wirable layouts which is of fundamental importance for what follows. It is based on the dual of the layout core. Let us call two layout edges that are incident to the same vertex and have a common face neighbored. For a layout $L$, define diagonal edges connecting certain vertices of core $(L)$ with vertices of core $(L)^{d}$. Precisely, for every pair of neighbored edges that belong to the same wire and whose common vertex is non-trivial, a diagonal edge is introduced conncting that common non-trivial vertex and the vertex of $\operatorname{cor} e(L)^{d}$ corresponding to the common face. See Figure 2 and Figure 3. For a vertex $v \in \operatorname{core}(L)^{d}$ denote $\operatorname{diag}(v)$ the number of diagonals incident to $v$. Then the extended degree of $v$, exdeg $(v)$, is the sum of the degree of $v$ (denoted $\operatorname{deg}(v)$ ), and the number of diagonal edges incident to $v$, i. e., $\operatorname{exdeg}(v):=\operatorname{deg}(v)+\operatorname{diag}(v)$. Now, $\operatorname{deg}(v)$ is equal to the number of edges on the face $F^{v}$ dual to $v$, which is again equal to the number of vertices on $F^{v}$. Thus, $\operatorname{exdeg}(v)=\left|F^{v}\right|+\operatorname{diag}(v)$. A vertex $v \in \operatorname{core}(L)^{d}$ is called even if $\operatorname{exdeg}(v)$ is an even number, otherwise $v$ is called odd.

Lemma 4. A layout $L$ is two-layer wirable if and only if

1. for each inner vertex of core $(L)^{d}$ the extended degree is even, and 


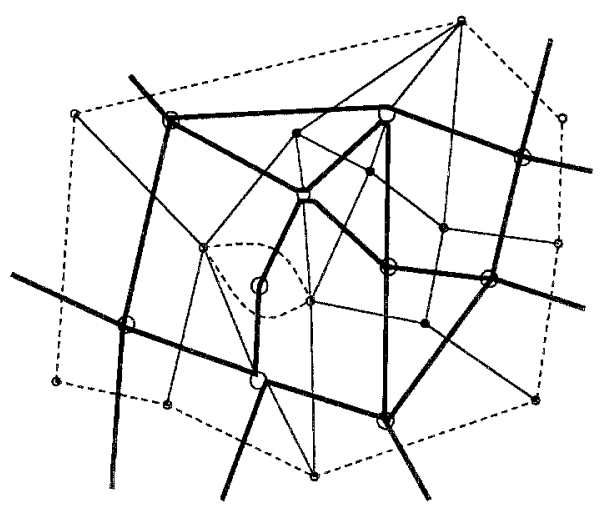

Fig. 3. Part of a layout core (bold), its dual and its diagonals (thin); the dashed edges are the boundary edges of the dual; inner vertices of the dual are black.

2. for each connected component of the boundary of core $(L)^{d}$ the sum of the extended degrees of the boundary vertices minus the number of boundary edges is even.

Proof. (Sketch) In a two-layer wiring, two neighbored edges incident to a common non-trivial vertex are assigned to different layers if and only if they belong to different wires. Thus, core $(L)$ is two-layer wirable if and only if for every face of core $(L)$ the number of vertices on that face incident to neighbored edges belonging to different wires is even.

For the first part of the proof, consider an inner vertex $v \in \operatorname{core}(L)^{d}$ dual to face $F^{v}$ of core $(L)$. Let $m(v)$ denote the number of vertices on $F^{v}$ incident to neighbored edges on $F^{v}$ belonging to different wires. Since an inner vertex $v$ is dual to a face $F^{v}$ containing only non-trivial vertices, $F^{v}$ is two-layer wirable if and only if $m(v)$ is even. Thus $F^{v}$ is two-layer wirable if and only if exdeg $(v)=$ $\left|F^{v}\right|+\operatorname{diag}(v)=m(v)+2 \operatorname{diag}(v)$ is even. The proof of the second part is similar, but more technical. It is omitted because of lack of space.

The general wiring theory developed now relies on the construction of an appropriate set of wire edges whose removal leaves a two-layer wirable layout. The declaration what "appropriate" means is our goal now. More precisely, for a $k$-layer wirable layout we give an equivalent characterization of a set of wire edges whose removal leaves a two-layer wirable layout. This characterization of a set of wire edges consists in giving forbidden patterns for its dual edges.

Definition 5. Consider a subset $R$ of wire edges of core $(L) . R$ is called a removal set of core $(L)$ if its removal leaves a two-layer wirable layout. The elements of $R$ are also called removal edges. Denote $R^{d}$ the set dual edges of $R$. A removal set $R$ is called legal if $R^{d}$ contains no cycle and no path connecting two vertices of the same connected component of the boundary of core $(L)^{d}$. 


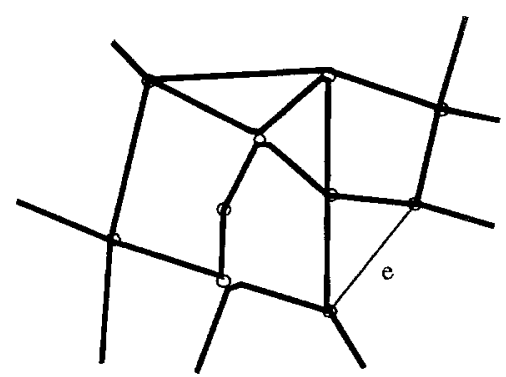

a)

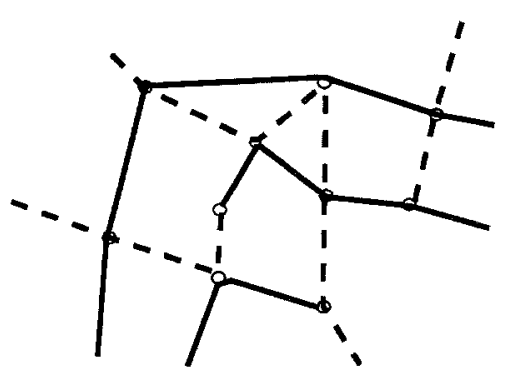

b)

Fig. 4. a) The layout $L$ from Figure 3. Edge $e$ forms a legal removal set (thin). b) A two-layer wiring of $L-\{e\}$

Consider a legal removal set $R$ of core $(L)$. If core $(L)$ is 2-edge-connected, the graph induced by core $(L)-R$ is connected. Thus it has a two-layer wiring that is unique up to the choice of the layers, say in layer bottom and layer top. Such a two-layer wiring induces a type classification of vertices $u$ and $v$ with respect to $\{u, v\}$, for every edge $\{u, v\} \in R$.

$$
\text { type }_{\{u, v\}}(v):= \begin{cases}1 & \text { if }\{u, v\} \text { is incident in } v \text { to a wire edge } \\ & \text { of the same wire in the top layer, or } \\ & \text { of a different wire in the bottom layer } \\ 2 & \text { otherwise. }\end{cases}
$$

Obviously, this classification is well defined. Observe, that for a vertex $v$ incident to edges $\{u, v\},\{w, v\} \in R$ that belong to different wires, type $\{u, v\}(v) \neq$ type $_{\{w, v\}}(v)$. Lemma 4 implies the following corollary.

Corollary 6. For a legal removal set $R$, the edges of $R^{d}$ form paths connecting odd vertices of core $(L)^{d}$, respectively an odd vertex to a boundary vertex of core $(L)^{d}$.

Since $R$ is legal, these paths form trees whose leaves are odd, respectively lie on the boundary. Note that two different paths containing a vertex of the same boundary component are considered to belong to the same tree.

Lemma 7. Let $R$ be a legal removal set of core $(L)$. For a type classification induced by a two-layer wiring of core $(L)-R, \operatorname{type}_{\{u, v\}}(v) \neq \operatorname{type}_{\{u, v\}}(u)$ for every edge $\{u, v\} \in R$.

Proof. (Sketch) A tree $T$ of edges of $R^{d}$ induces a cycle of wire edges not in $R$, i. e., the cycle of edges of core $(L)$ around $T$. More precisely, every inner vertex of core $(L)$ belonging to $T$ corresponds to its dual cycle in core $(L)$, and every boundary vertex of core $(L)$ belonging to $T$ corresponds to the cycle in core $(L)$ 
around the corresponding boundary component. Then the union of these cycles minus the edges of $R$ form the cycle induced by $T$.

In a two-layer wiring of core $(L)-R$, such a cycle must contain an even number of layer changes. Now, consider an edge $\{u, v\} \in R$ dual to an edge of $T$. Assume type $\{u, v\}(v)=$ type $_{\{u, v\}}(u)$. Then for all edges $\{x, y\} \in R$ dual to an edge of $T$ we have type $\{x, y\}(x)=$ type $_{\{x, y\}}(y)$. But for an edge of $R$ dual to an edge incident to a leaf of $T$, the type of the two end vertices must be different with respect to that edge. This is a contradiction.

Lemma 7 guarantees that we can define an orientation on the edges of $R$.

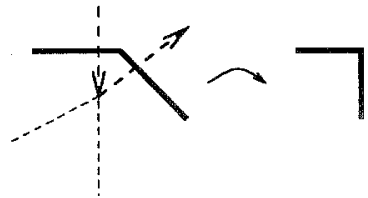

a)

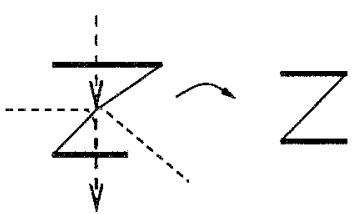

b)

Fig. 5. Removal sets that do not induce 3-layer wirability and the corresponding forbidden patterns. a) Pattern corresponding to neigboring edges. b) Pattern corresponding to edges of the same face that are not neigboring and are both incident to a diagonal ending at the same vertex.

Definition 8. An edge $\{u, v\} \in R$ is oriented from $u \rightarrow v$ if $\operatorname{type}_{\{u, v\}}(u)=1$ and $\operatorname{type}_{\{u, v\}}(v)=2$. Let $R^{\rightarrow}$ denote the directed graph, called layer graph, induced by $R$ and this orientation.

Obviously, two adjacent arcs of $R^{\rightarrow}$ that belong to the same wire are oriented towards each other. So, two subsequent edges on a directed path in $R \rightarrow$ must belong to different wires. The length of a directed path is defined as the number of edges on that path. Now we are ready to prove the main theorem of this section.

Theorem 9. A layout $L$ is wirable in $k$ layers if and only if there exists a legal removal set $R$ such that the length of any directed path in $R \rightarrow$ is at most $k-2$.

Proof. " $\Rightarrow$ " Consider a wiring of core $(L)$ in layer $L_{i}, 0 \leq i \leq k-1$. Let $R$ be the set of all wire edges wired in layer $L_{i}, 0 \leq i \leq k-1$, and $R^{\rightarrow}$ the corresponding directed layer graph. For a directed path in $R^{\rightarrow}$, two subsequent arcs $(u, v)$ and $(v, w)$ belong to different wires and thus must be wired in different layers. Because of the orientation, the layer of $(u, v)$ must be below the layer of $(v, w)$. Consequently, the length of any directed path in $R \rightarrow$ is at most $k-2$. 


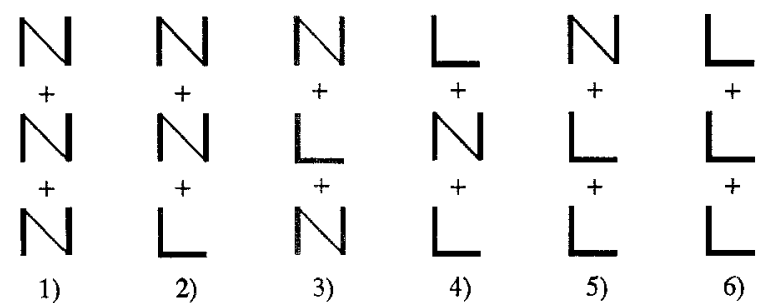

a)

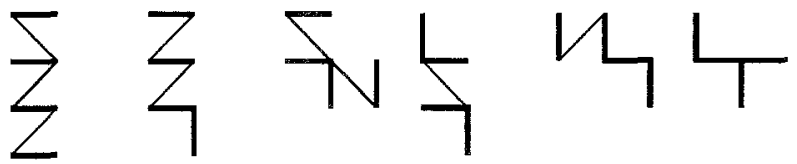

b)

Fig. 6. a) Combinations of forbidden patterns for 3-layer wirability that lead to forbidden patterns for 5-layer wirability; b) examples of forbidden patterns for 5-layer wirability .

" $\Leftarrow$ " Assume there exists a legal removal set $R$ such that the length of every directed path in $R^{\rightarrow}$ is at most $k-2$. For an edge $(u, v) \in R^{\rightarrow}$ denote $l_{\max }(u, v)$ the maximum length of a directed path in $R^{\rightarrow}$ terminating with $(u, v)$. A wiring of $\operatorname{core}(L)$ in $k$ layers $L_{i}, 0 \leq i \leq k-1$, is constructed as follows.

$$
\text { layer }(u, v)= \begin{cases}L_{0} \text { resp. } L_{k-1}, & \text { if }(u, v) \notin R^{\rightarrow} ; \\ L_{i}, 1 \leq i \leq k-2, & \text { if }(u, v) \in R^{\rightarrow} \text { and } l_{\text {max }}(u, v)=i-1 .\end{cases}
$$

The assignment of $(u, v)$ to $L_{0}$ resp. $L_{k-1}$ is according to a fixed two-layer wiring of $\operatorname{core}(L)-R$.

Remark The proof of Theorem 9 induces an algorithm to construct a $k$-layer wiring from a legal removal set $R$ where the length of any directed path in $R \rightarrow$ is at most $k-2$. This algorithm can be easily implemented to run in time linear in the size of the layout.

A characterization of $k$-layer wirability for $k>2$ in terms of forbidden patterns in the dual $R^{d}$ of a legal removal set $R$ is now easily derived from Theorem 9 .

Lemma 10. A layout $L$ is wirable in three layers if and only if there exists a legal removal set $R$ such that $R^{d}$ contains none of the patterns shown in Figure 5.

Proof. core $(L)$ is wirable in three layers if and only if there exists a legal removal set $R$ such that $R^{\rightarrow}$ contains no directed path of length two. For a directed path in $R \rightarrow$ two subsequent edges must belong to different wires. These two edges meet 
at a non-trivial vertex, say $v$. In case the two corresponding dual edges in $R^{d}$ are neigboring in $v$, they form a pattern of type a) shown in Figure 5. Otherwise they form a pattern of type b) shown in Figure 5 , i. e., the two corresponding dual edges in $R^{d}$ belong to the same face, but are not neigboring in $v$ and are each incident to a diagonal ending at $v$.

Lemma 10 can be easily extended to giving forbidden patterns for wirability in $k \geq 4$ layers. From the patterns shown in Figure 5 we just have to generate patterns dual to directed paths of length $k-1$ in the layer graph. Forbidden patterns for wirability in four layers are all possible combinations of two forbidden patterns for wirability in three layers, where two patterns are combined by identifying two edges. Accordingly, forbidden patterns for wirability in five layers are all possible combinations of three forbidden patterns for wirability in three layers. See Figure 6.

\section{Algorithms}

In this section we prove that every layout in a tri-hexagonal grid and every layout in a tri-square-hexagonal grid is wirable in five layers. We present algorithms that construct a removal set for such a layout satisfying Theorem 9 for $k=5$. That is, the dual $R^{d}$ corresponding to the constructed removal set $R$ contains none of the patterns illustrated in Figure 6.

\subsection{Layouts in Tri-Hexagonal Grid Graphs}

A tri-hexagonal grid graph is a grid consisting of grid lines of three different directions, the horizontal direction and two diagonal directions. In every grid point, lines of two different directions meet, either one horizontal and one diagonal line, or two diagonal lines. Consequently, every vertex has degree four. See Figure 7 a) for a tri-hexagonal grid and its dual.

The algorithm to construct a legal removal set $R$ for a layout $L$ in a trihexagonal grid works as follows. Firstly, all vertices of the layout are considered to be non-trivial. Then obviously, 5-layer wirability of this layout induces 5-layer wirability of the original layout. The dual of the layout is scanned "row-wise" from bottom to top, and from left to right. For every vertex of the dual of the layout, its extended degree and its vertex class is considered. That is, the vertex set of the grid dual to a tri-hexagonal grid is partitioned into three different classes: vertices of degree six (class 1), vertices of degree three incident to an up-going vertical edge and two diagonal edges (class 2), and vertices of degree three incident to an down-going vertical edge and two diagonal edges (class 3 ). See Figure 8. Now alternatingly, in every second row only vertices of class 1 and class 2 are visited, or only vertices of class 3 . Depending on the extended degree and the class of the visited vertex, an incident edge is added to $R^{d}$ in such a way, that $\operatorname{exdeg}(v)$ is even for all vertices $v \in L^{d}-R^{d}$, and $R^{d}$ contains no forbidden pattern for 5-layer wirability. 


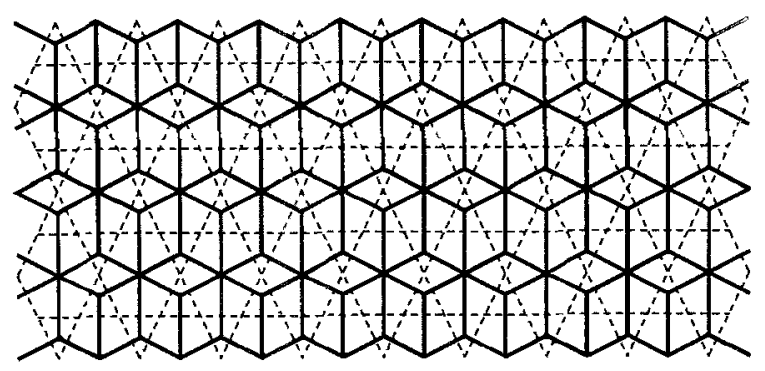

a)

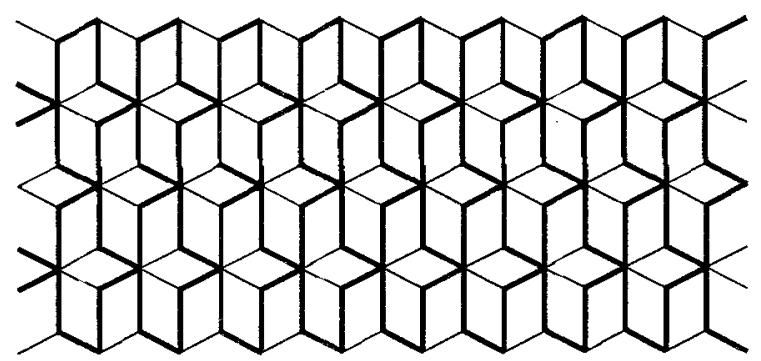

b)

Fig. 7. a) A tri-hexagonal grid graph (dashed) and its dual graph (bold). b) Dual edges that might be added to $R^{d}$ by the algorithm (bold).

\section{Algorithm 1 Tri-Hexa}

Input: The dual $L^{d}$ of a layout $L$ in a tri-hexagonal grid.

Output: A subset $R^{d}$ of $L^{d}$ such that $\operatorname{exdeg}(v)$ is even for all vertices $v \in L^{d}-R^{d}$ and $R^{d}$ contains no forbidden pattern for 5 -layer wirability.

\section{begin}

$R^{d}:=\emptyset$

for all rows do

for all vertices $v$ of one row do if exdeg $(v)$ is odd in $L^{d}$ and $v$ belongs to class 2, then $R^{d}:= \begin{cases}R^{d}+\epsilon_{\text {right }}, & \text { if right successor is odd; } \\ R^{d}+e_{u p} & \text { otherwise. }\end{cases}$ belongs to class 1 , then $R^{d}:=R^{d}+e_{u p}$ belongs to class 3 , then $R^{d}:=R^{d}+e_{\text {right }}$ $L^{d}:=L^{d}-R^{d}$ 


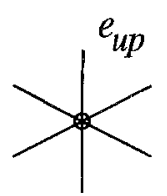

1)

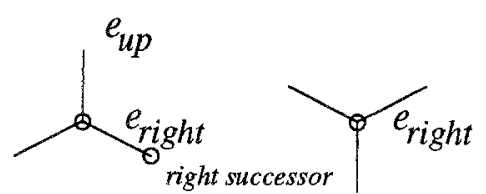

2)

Fig. 8. The three different classes of vertices in the grid dual to a tri-hexagonal grid.

It is easy to prove that Algorithm 1 considers only those edges of $L^{d}$ shown in Figure $7 \mathrm{~b}$ ). Then the following theorem can be proved by a detailed case analysis.

Theorem 11. For a layout $L$ in a tri-hexagonal grid, Algorithm 1 constructs a subset $R^{d}$ of $L^{d}$ that contains no forbidden pattern for 5-layer wirability. The running time is linear in the size of the layout.

Proof is omitted.

From a legal removal set determined by Algorithm 1, a 5-layer wiring of a layout in a tri-hexagonal grid can be constructed in linear time as well according to Theorem 9 .

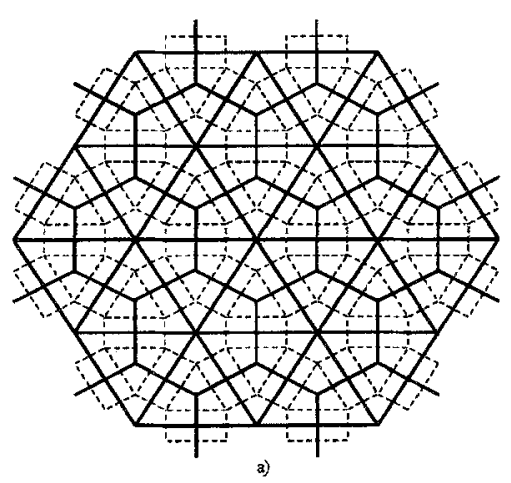

Fig. 9. a) A tri-square-hexagonal grid graph (dashed) and its dual graph (bold). b) Dual edges that might be added to $R^{d}$ by the algorithm (bold).

\subsection{Layouts in Tri-Square-Hexagonal Grid Graphs}

A tri-square-hexagonal grid graph is the dual graph of the union of two grids, a hexagonal grid and the dual of a hexagonal grid. Thus it contains only vertices 
of degree four. Its faces are triangles, squares and hexagons. See Figure 9 for a tri-square-hexagonal grid and its dual.

The algorithm to construct a legal removal set $R$ for a layout $L$ in a tri-squarehexagonal grid again scans the dual of the layout "row-wise" from bottom to top and from left to right. The vertices of the grid dual to a tri-square-hexagonal grid are partitioned into six different classes: vertices of degree six (class 1), three different classes of vertices of degree four (class 2,3 and 4), and two different classes of vertices of degree three (class 5 and 6). See Figure 10.

Alternatingly, in every second row only vertices of class 1 and class 2 are visited, or only vertices of class $3,4,5$ and 6 . Depending on the extended degree and the class of the visited vertex, an edge is added to $R^{d}$ in such a way that $\operatorname{exdeg}(v)$ is even for all vertices $v \in L^{d}-R^{d}$, and $R^{d}$ contains no forbidden pattern for 5-layer wirability.

\section{Algorithm 2 Tri-Square-Hexa}

Input: The dual $L^{d}$ of a layout $L$ in a tri-square-hexagonal grid.

Output: $A$ subset $R^{d}$ of $L^{d}$ such that exdeg $(v)$ is even for all vertices $v \in$ $L^{d}-R^{d}$, and $R^{d}$ contains no forbidden pattern for 5-layer wirability.

\section{begin \\ $R^{d}:=\emptyset$}

for all rows do

for all vertices $v$ of one row do

if exdeg $(v)$ is odd in $L^{d}$ and $v$

belongs to class 1 , then $R^{d}:= \begin{cases}R^{d}+e_{\text {right }}, & \text { if right successor is odd; } \\ R^{d}+e_{u p} & \text { otherwise. }\end{cases}$

belongs to class 2 , then $R^{d}:=R^{d}+e_{u p}$

belongs to class 3 , then $R^{d}:=R^{d}+e_{\text {right }-u p}$

belongs to class 4 , then $R^{d}:=R^{d}+e_{\text {right-up }}$

belongs to class 5 , then $R^{d}:=R^{d}+e_{\text {right }}$

belongs to class 6, then $R^{d}:= \begin{cases}R^{d}+e_{\text {right }}, & \text { if right successor is odd; } \\ R^{d}+e_{u p} & \text { otherwise. }\end{cases}$ $L^{d}:=L^{d}-R^{d}$

end

It can be shown that Algorithm 2 considers only those edges of $L^{d}$ shown in Figure $9 \mathrm{~b}$ ). Then by a detailed case analysis, the following theorem can be proved.

Theorem 12. For a layout $L$ in a tri-square-hexagonal grid Algorithm 2 constructs a subset $R^{d}$ of $L^{d}$ that contains no forbidden patterns for 5-layer wirability. The running time is linear in the size of the layout. 


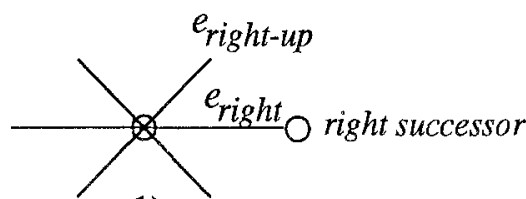

1)

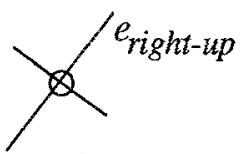

3)

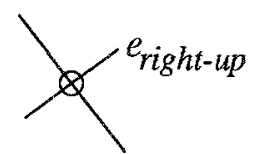

4)

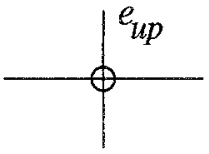

2)

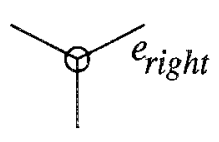

5)

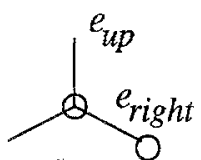

6)

right successor

Fig. 10. The six different classes of vertices in the grid dual to a tri-square-hexagonal grid.

Proof is omitted.

From a legal removal set determined by Algorithm 2, a 5-layer wiring of a layout in a tri-square-hexagonal grid can be constructed in linear time as well according to Theorem 9.

\section{Concluding Remarks}

We presented a general approach to the problem of wiring edge-disjoint layouts. Equivalent conditions for the $k$-layer wirability of an edge-disjoint layout where at most two wires meet in a vertex are given. Based on these conditions, we obtain linear-time algorithms to wire every layout in a tri-hexagonal grid and every layout in a tri-square-hexagonal grid using at most five layers. Our approach generalizes the framework introduced in [8]. There, equivalent conditions for the $k$-layer wirability of an edge-disjoint layout in a square grid are given. These conditions are based on a legal partition of the layout grid into a twocolorable map. A legal partition is characterized by "forbidden patterns" for the partition lines. The two color regions of the partition correspond to regions where horizontal edges are wired above vertical edges, resp. to regions where vertical edges are wired above horizontal edges. This framework is not applicable to more general grids resp. planar layout graphs, since there we can have layout edges of more than two different directions. The equivalent conditions for $k$-layer wirability developed in this paper are based on the characterization of legal removal sets. This leads to "forbidden pattern" as well, which are quite similar to the forbidden patterns given in [8]. Overall, our approach delivers a more general interpretation of the framework introduced there.

Acknowledgements We thank Majid Sarrafzadeh for drawing our attention to the layer assignment problem for general edge-disjoint layouts. 


\section{References}

1. M. Brady and D. Brown: VLSI routing: Four layers suffice. In F. Preparata, editor, Advances in Computer Research, VOL 2: VLSI Theory, JAI Press Inc. (1984) 245257

2. M. Brady and M. Sarrafzadeh: Stretching a knock-knee layout for multilayer wiring. IEEE Transactions on Computers, C-39 (1990) 148-152

3. G. Di Battista, P. Eades, R. Tamassia, and I. G. Tollis: Algorithms for Drawing Graphs: an Annotated Bibliography. Computational Geometry 4 (1994) 235-282

4. T. F. Gonzales and S. Zheng: Simple three-layer channel routing algorithms. In J. H. Reif, editor, Proceedings Aegean Workshop on Computing. Springer-Verlag, Lecture Notes in Computer Science, vol. 319 (1988) 237-246

5. M. Kaufmann and P. Molitor: Minimal stretching of a layout to ensure 2-layer wirability. INTEGRATION The VLSI Journal, 12 (1991) 339-352

6. R. Kuchem, D. Wagner, and F. Wagner: Optimizing area for three-layer channel routing. Algorithmica, 15 (1996) 495-519

7. W. Lipski, Jr: On the structure of three-layer wirable layouts. In F. Preparata, editor, Advances in Computer Research, VOL 2: VLSI Theory, JAI Press Inc. (1984) 231-243

8. W. Lipski, Jr and F. P. Preparata: A unified approach to layout wirability. Math. Systems Theory, 19 (1987) 189-203

9. R. H. Möhring, D. Wagner, and F. Wagner: VLSI Network Design: A survey. In M. Ball, T. Magnanti, C. Monma, and G. Nemhauser, editors, Handbooks in Operations Research/Management Science, Volume on Networks, North-Holland, (1995) $625-712$

10. P. Molitor: A survey on wiring. J. Inform. Process. Cybernet. EIK, 27 (1991) 3-19

11. H. Ripphausen-Lipa, D. Wagner, and K. Weihe: Efficient algorithms for disjoint paths in planar graphs. In W. Cook, L. Lovász, and P. Seymour, editors, DIMACS, Center for Discr. Math. and Comp. Sc., 20, Springer-Verlag, Berlin (1995) 295-354

12. M. Sarrafzadeh, D. Wagner, F. Wagner, and K. Weihe: Wiring knock-knee layouts: a global approach. IEEE Transactions on Computers 43 (1994) 581-589

13. I. G. Tollis: Wiring Layouts in the Tri-Hexagonal Grid. Intern. J. Computer Math. 37 (1990) 161-171

14. I. G. Tollis: A New Approach to Wiring Layouts. IEEE Transactions on ComputerAided Design of Integrated Circuits and Systems 10 (1991) 1392-1400

15. I. G. Tollis: Wiring in uniform grids and two-colorable maps. INTEGRATION The VLSI Journal 12 (1991) 189-210

16. D. Wagner: Optimal routing through dense channels. Int. J. on Comp. Geom. and Appl. 3 (1993) 269-289 